\title{
Umsetzung der S3-Leitlinie zur Prävention, Diagnostik, Therapie und Nachsorge des Lungenkarzinoms im Land Berlin*
}

\section{Implementation of the German Guideline for the Prevention, Diagnosis, Treatment, and Follow-up of Lung Cancer in the Federal State of Berlin}

Autoren

Institute
T. Blum ${ }^{1}$, N. Schönfeld ${ }^{1}$, G. Goeckenjan ${ }^{2}$, A. Jagota ${ }^{3}$

${ }^{1}$ Klinik für Pneumologie, Lungenklinik Heckeshorn, HELIOS Klinikum Emil von Behring, Berlin

${ }^{2}$ Kassel

${ }^{3}$ Tumorzentrum Berlin e.V. eingereicht $\quad 30.7 .2012$ akzeptiert nach Revision 10. 11. 2012

\section{Bibliografie}

Dol http://dx.doi.org/ 10.1055/s-0032-1325981 Online-Publikation: 18.12.2012

Pneumologie 2013; 67: 118-122 (c) Georg Thieme Verlag KG Stuttgart · New York ISSN 0934-8387

Korrespondenzadresse Dr. Torsten Blum, M.B.A. Klinik für Pneumologie Lungenklinik Heckeshorn HELIOS Klinikum Emil von Behring Walterhöferstr. 11 14165 Berlin torsten-gerriet.blum@helioskliniken.de

\section{Zusammenfassung \\ V}

Hintergrund: In Februar 2010 wurde die deutschsprachige S3-Leitlinie zur Diagnostik und Therapie des Lungenkarzinoms publiziert. Erstmals in Deutschland wurde im Land Berlin die Umsetzung relevanter Leitlinienempfehlungen überprüft.

Methoden: Anhand der Qualitätsindikatoren der S3-Leitlinie wurde ein Fragebogen erstellt, der die Anwendung ausgewählter diagnostischer und therapeutischer Konzepte zum Inhalt hatte. Der Bogen wurde an alle potenziellen fachspezifischen Behandler (ambulant und stationär) im Land Berlin versendet.

Ergebnisse: Bei guter Rücklaufquote (39,8\%) v.a. aus dem spezialisierten stationären Sektor zeigte sich die größte Leitlinientreue bei den stationären pneumologischen Einrichtungen, gefolgt von onkologischen.

Schlussfolgerungen: Bei methodisch eingeschränkter Aussagekraft, wie sie in der Natur einer offenen Befragung liegt, zeichnete sich ein Trend ab, dass die Leitlinie mit abnehmendem Spezialisierungsgrad schlechter umgesetzt wird.

\section{Einleitung}

$\nabla$

Das Krankheitsbild Lungenkrebs erfordert sowohl in Diagnostik als auch Therapie eine hohe interdisziplinäre Kompetenz. Die morphologische und biologische Heterogenität der Tumoren sowie die besonders hohe Komorbiditätslast erfordern ein optimales Zusammenspiel vor allem zwischen Pneumologen, Thoraxchirurgen und Strahlentherapeuten. Viele, aber nicht alle Lungenkrebspatienten werden in pneumologisch-thoraxchirurgischen Einrichtungen versorgt. Zur Gewährleistung einer möglichst flächendeckenden Behandlungsqualität haben die Deutsche Gesellschaft für Pneumologie und Beatmungsmedizin,

\section{Abstract \\ $\nabla$}

Background: In February 2010, the German guideline for the Prevention, Diagnosis and Treatment of Lung Cancer has been published. For the first time in Germany, the implementation of relevant guideline recommendations has been assessed in the federal state of Berlin.

Methods: Based on the quality indicators of the guideline, a questionnaire was designed which aimed at the application of selected diagnostic and therapeutic concepts. This questionnaire was sent out to all outpatient and inpatient units in the federal state of Berlin being presumably involved in lung cancer care.

Results: Overall response rate was good (39.8\%), especially from the addressed inpatient units. Inpatient pneumology units showed the highest application rates of guideline recommendations, followed by oncology units.

Conclusions: Taking the methodologically reduced significance of a survey design into account, this study determined a negative trend of decreasing guideline implementation in units with a lesser grade of specialisation in terms of lung cancer care.

die Deutsche Gesellschaft für Thoraxchirurgie und die Deutsche Krebsgesellschaft zusammen mit weiteren Fachverbänden eine S3-Leitlinie für das Krankheitsbild erstellt [1].

\footnotetext{
* für die Projektgruppe Lungenkarzinom, Tumorzentrum Berlin e.V. (Geschäftsführerin: Frau Dr. P. H. Dipl.-Soz. Anita Jagota):

Harun Badakhshi, Barbara Baysal, Jörg Beyer, Daniel Binder, Maike de Wit, Stephan Eggeling, Matthias Franz, Christian Gogoll, Christian Grah, Christian Grohé, Marcel Haug, Ulrike Höller, Dirk Kaiser, Mi-Ri Kim, Jens Kollmeier, Matthias Krüll, Christel Lauer, Gunda Leschber, Uta Liebers, Ronald Lützenberg, Jens Neudecker, Wulf Pankow, Jens-C. Rückert, Alexander Schmittel, Paul Schneider, Antje Tessmer, Martin Utzig, Julia Vlad, Natalia Wiener, Christian Witt.
} 
Qualitätssicherung Bereich Pneumologie- Onkologie

Werden bei Ihnen folgende Diagnostik- und Therapiemöglichkeiten bei Patienten mit Lungenkrebs genutzt?

\begin{tabular}{|c|c|c|}
\hline \multirow{2}{*}{$\begin{array}{l}\text { Diagnostik } \\
\text { bei Patienten mit nichtkleinzelligem Lungenkarzinom und auf der CT Thorax-Untersuchung basierender kurativer Therapieintention: }\end{array}$} & Ja & Nein \\
\hline & & \\
\hline - FDG-PET & & \\
\hline - Mediastinoskopie & & \\
\hline - transbronchialer Endosonografie & & \\
\hline Therapie & & \\
\hline $\begin{array}{l}\text { - Adjuvante Chemotherapie bei Patienten mit nichtkleinzelligem Lungenkarzinom nach R0-Resektion und systematischer } \\
\text { Lymphknotendissektion in den Stadien II bis IIIA1/2. }\end{array}$ & & \\
\hline - Kombinierte Radiochemotherapie im Stadium IIIA4/IIIB beim nichtkleinzelligen Lungenkarzinom. & & \\
\hline $\begin{array}{l}\text { - Cisplatin-basierte Kombinationschemotherapie bei Patienten mit nichtkleinzelligem Lungenkarzinom in den Stadien } \\
\text { IIIB (Pleuraerguss) und IV. }\end{array}$ & & \\
\hline - Zweitlinienchemotherapie bei Patienten mit nichtkleinzelligem Lungenkarzinom und Progress nach primärer Chemotherapie. & & \\
\hline $\begin{array}{l}\text { - Kombinierte Radiochemotherapie bei Patienten mit kleinzelligem Lungenkarzinom in den bestrahlungsfähigen Stadien } \\
\text { IIB(T3) - IIIB (TNM: cT1/2 N2 - } 3 \text { M0, cT3/4 N0 - 3 M0). }\end{array}$ & & \\
\hline $\begin{array}{l}\text { - Prophylaktische Radiatio des Hirnschädels bei Patienten mit kleinzelligem Lungenkarzinom und Remission nach Abschluss der } \\
\text { Primärtherapie. }\end{array}$ & & \\
\hline
\end{tabular}

Führen Sie eine Erfassung der Lebensqualität bei Patienten mit Lungenkrebs durch?

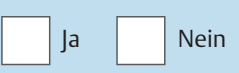

Falls ja,

- mit welchem Instrument(en) (z.B. EORTC-QLQ-C30), SF-6)?

- zu welchen Zeitpunkten (z.B. Erstdiagnose, nach 6 Monaten, nach Operation etc.)?

Abb.1 Fragebogen auf der Grundlage der Qualitätsindikatoren 2 - 8 der S3-Leitlinie zur Prävention, Diagnostik, Therapie und Nachsorge des Lungenkarzinoms.

Mehr als ein Jahr nach Veröffentlichung dieser Leitlinie stellt sich die Frage nach deren Umsetzung sowohl in spezialisierten als auch nicht spezialisierten Einrichtungen. Durch die jährlichen Veröffentlichungen zur Häufigkeit der operativen Behandlung des Lungenkrebses in den Berliner Krankenhäusern gibt es zumindest Hinweise für die Verteilung der Patienten auf die Einrichtungen verschiedener Art [2]. Ferner existiert eine Arbeit, die eine Übersicht über Versorgungsstrukturen bei der Behandlung von Lungenkrebspatienten bietet [3]. Darüber hinausgehende Daten zur Behandlungsqualität selbst wurden aber bisher in Deutschland noch nicht veröffentlicht. Die vorliegende Studie berichtet über eine erste Umfrage zur Qualitätsicherung anhand der Qualitätsindikatoren in der Leitlinie im Land Berlin.

\section{Material und Methoden}

Die Querschnittstudie widmete sich dem Thema Qualitätssicherung im Bereich der konservativen Lungenkarzinom-Behandlung innerhalb der potenziell an der Versorgung teilnehmenden stationären und ambulanten Versorgungseinrichtungen im Land Berlin. Als Basis für die empirische Erhebung diente ein Fragebogen, der Fragen zu den Bereichen Diagnostik und Therapie des Lungenkarzinoms, basierend auf den in der S3-Leitlinie formulierten Qualitätsindikatoren 2-8 [1], sowie zur Erfassung der Lebensqualität enthielt (s. $\odot$ Abb.1). Der Fragebogen wurde am 9.11.2010 postalisch an alle stationären pneumologischen, onko- logischen und allgemeininternistischen Einrichtungen, ferner an alle pneumologischen und onkologischen Schwerpunktpraxen im Land Berlin versendet. Die jeweiligen Verteilerlisten wurden im Vorfeld mittels Online-Abfragen von spezifischen Adressdateien der Kassenärztlichen Vereinigung Berlin [4] bzw. des Berliner Krankenhausverzeichnisses [5] generiert.

Die Befragung wurde Ende Januar 2011 geschlossen. Die statistische Auswertung der Ergebnisse erfolgte im Februar $2011 \mathrm{mit}$ Hilfe des Statistikprogramms SPSS 18.0 (SPSS Inc., Chicago, IL, USA).

Am 4.4.2011 erfolgte - nach vorhergehender schriftlicher Einladung aller zuvor angeschriebenen Einrichtungen - ein Audit zur Ergebnispräsentation, bei der alle Teilnehmer die Gelegenheit hatten, die Ergebnisse zu bewerten und zu kommentieren.

Tab. 1 Übersicht über angeschriebene Versorgungseinrichtungen sowie deren jeweilige Rücklaufquoten.

\begin{tabular}{|lcc|}
\hline Einrichtungsart & Angeschrieben & Rücklauf \\
\hline Kliniken für Innere Medizin (Allgemein) & 21 & $7(33,3 \%)$ \\
\hline Kliniken für Innere Medizin (Onkologie) & 9 & $8(88,9 \%)$ \\
\hline pneumologische Kliniken & 7 & $7(100,0 \%)$ \\
\hline onkologische Schwerpunktpraxen & 30 & $13(43,3 \%)$ \\
\hline Pneumologiepraxen & 46 & $10(21,7 \%)$ \\
\hline Gesamtergebnis & 113 & $45(39,8 \%)$
\end{tabular}


Tab.2 Umfrageergebnisse zu den Bereichen Diagnostik und Therapie des Lungenkarzinoms, basierend auf den in der S3-Leitlinie formulierten Qualitätsindikatoren $2-8$.

\begin{tabular}{|c|c|c|c|c|c|}
\hline \multirow[b]{2}{*}{ Qualitätsindikator } & \multicolumn{3}{|l|}{ stationäre Einrichtungen } & \multicolumn{2}{|c|}{ Schwerpunktpraxen } \\
\hline & $\begin{array}{l}\text { allgemein-internistisch } \\
n=7\end{array}$ & $\begin{array}{l}\text { onkologisch } \\
n=8\end{array}$ & $\begin{array}{l}\text { pneumologisch } \\
n=7\end{array}$ & $\begin{array}{l}\text { onkologisch } \\
n=13\end{array}$ & $\begin{array}{l}\text { pneumologisch } \\
n=10\end{array}$ \\
\hline \multicolumn{6}{|c|}{$\begin{array}{l}\text { FDG-PET bei Patienten mit nichtklein- } \\
\text { zelligem Lungenkarzinom und auf der } \\
\text { CT Thorax-Untersuchung basierender } \\
\text { kurativer Therapieintention }\end{array}$} \\
\hline - ja & $3(42,9 \%)$ & $6(75,0 \%)$ & $7(100,0 \%)$ & $10(76,9 \%)$ & $8(80,0 \%)$ \\
\hline - nein & $4(57,1 \%)$ & $2(25,0 \%)$ & $0(0,0 \%)$ & $3(23,1 \%)$ & $2(20,0 \%)$ \\
\hline \multicolumn{6}{|c|}{$\begin{array}{l}\text { Mediastinoskopie bei Patienten mit nicht- } \\
\text { kleinzelligem Lungenkarzinom und auf } \\
\text { der CT Thorax-Untersuchung basierender } \\
\text { kurativer Therapieintention }\end{array}$} \\
\hline- ja & $3(42,9 \%)$ & $5(62,5 \%)$ & $7(100,0 \%)$ & $9(69,2 \%)$ & $7(70,0 \%)$ \\
\hline - nein & $4(57,1 \%)$ & $3(37,5 \%)$ & $0(0,0 \%)$ & $3(23,1 \%)$ & $2(20,0 \%)$ \\
\hline - keine Antwort & $0(0 \%)$ & $0(0 \%)$ & $0(0 \%)$ & $1(7,7 \%)$ & $1(10,0 \%)$ \\
\hline \multicolumn{6}{|c|}{$\begin{array}{l}\text { EBUS bei Patienten mit nichtkleinzelligem } \\
\text { Lungenkarzinom und auf der CT Thorax- } \\
\text { Untersuchung basierender kurativer } \\
\text { Therapieintention }\end{array}$} \\
\hline - ja & $1(14,3 \%)$ & $5(62,5 \%)$ & $7(100,0 \%)$ & $10(76,9 \%)$ & $7(70,0 \%)$ \\
\hline- nein & $6(85,7 \%)$ & $3(37,5 \%)$ & $0(0,0 \%)$ & $3(23,1 \%)$ & $2(20,0 \%)$ \\
\hline - keine Antwort & $0(0 \%)$ & $0(0 \%)$ & $0(0 \%)$ & $0(0 \%)$ & $1(10,0 \%)$ \\
\hline \multicolumn{6}{|c|}{$\begin{array}{l}\text { Adjuvante Chemotherapie bei Pat. mit } \\
\text { nichtkleinzelligem Lungenkarzinom nach } \\
\text { R0-Resektion und systematischer Lymph- } \\
\text { knotendissektion in den Stadien II bis IIIA1/2 }\end{array}$} \\
\hline- ja & $4(57,1 \%)$ & $6(75,0 \%)$ & $7(100,0 \%)$ & $11(84,6 \%)$ & $8(80,0 \%)$ \\
\hline - nein & $3(42,9 \%)$ & $2(25,0 \%)$ & $0(0,0 \%)$ & $2(15,4 \%)$ & $2(20,0 \%)$ \\
\hline \multicolumn{6}{|c|}{$\begin{array}{l}\text { Kombinierte Radiochemotherapie im } \\
\text { Stadium IIIA4/IIIB beim nichtkleinzelligen } \\
\text { Lungenkarzinom }\end{array}$} \\
\hline- ja & $4(57,1 \%)$ & $7(87,5 \%)$ & $7(100,0 \%)$ & $12(92,3 \%)$ & $8(80,0 \%)$ \\
\hline - nein & $3(42,9 \%)$ & $1(12,5 \%)$ & $0(0,0 \%)$ & $1(7,7 \%)$ & $2(20,0 \%)$ \\
\hline \multicolumn{6}{|c|}{$\begin{array}{l}\text { Cisplatin-basierte Kombinationschemo- } \\
\text { therapie bei Pat. mit nichtkleinzelligem } \\
\text { Lungenkarzinom in den Stadien IIIB } \\
\text { (Pleuraerguss) und IV }\end{array}$} \\
\hline - ja & $4(57,1 \%)$ & $6(75,0 \%)$ & $7(100,0 \%)$ & $12(92,3 \%)$ & $8(80,0 \%)$ \\
\hline - nein & $3(42,9 \%)$ & $2(25,0 \%)$ & $0(0,0 \%)$ & $1(7,7 \%))$ & $2(20,0 \%)$ \\
\hline \multicolumn{6}{|c|}{$\begin{array}{l}\text { Zweitlinienchemotherapie bei Pat. mit } \\
\text { nichtkleinzelligem Lungenkarzinom und } \\
\text { Progress nach primärer Chemotherapie }\end{array}$} \\
\hline$-\mathrm{ja}$ & $4(57,1 \%)$ & $7(87,5 \%)$ & $7(100,0 \%)$ & $12(92,3 \%)$ & $8(80,0 \%)$ \\
\hline - nein & $3(42,9 \%)$ & $1(12,5 \%)$ & $0(0,0 \%)$ & $1(7,7 \%)$ & $2(20,0 \%)$ \\
\hline \multicolumn{6}{|c|}{$\begin{array}{l}\text { Kombinierte Radiochemotherapie bei Pat. } \\
\text { mit kleinzelligem Lungenkarzinom in den } \\
\text { bestrahlungsfähigen Stadien IIB[T3] - IIIB } \\
\text { (TNM: cT1/2 N2 - 3 M0, cT3/4 N0-3 M0) }\end{array}$} \\
\hline- ja & $4(57,1 \%)$ & $7(87,5 \%)$ & $7(100,0 \%)$ & $12(92,3 \%)$ & $8(80,0 \%)$ \\
\hline - nein & $3(42,9 \%)$ & $1(12,5 \%)$ & $0(0,0 \%)$ & $1(7,7 \%)$ & $2(20,0 \%)$ \\
\hline \multicolumn{6}{|c|}{$\begin{array}{l}\text { Prophylaktische Radiatio des Hirnschädels } \\
\text { bei Pat. mit kleinzelligem Lungenkarzinom } \\
\text { und Remission nach Abschluss der Primär- } \\
\text { therapie }\end{array}$} \\
\hline - ja & $4(57,1 \%)$ & $7(87,5 \%)$ & $7(100,0 \%)$ & $10(76,9 \%)$ & $7(70,0 \%)$ \\
\hline - nein & $3(42,9 \%)$ & $1(12,5 \%)$ & $0(0,0 \%)$ & $3(23,1 \%)$ & $3(30,0 \%)$ \\
\hline
\end{tabular}


Tab.3 Umfrageergebnisse zu dem Fragekomplex Lebensqualitätmessung.

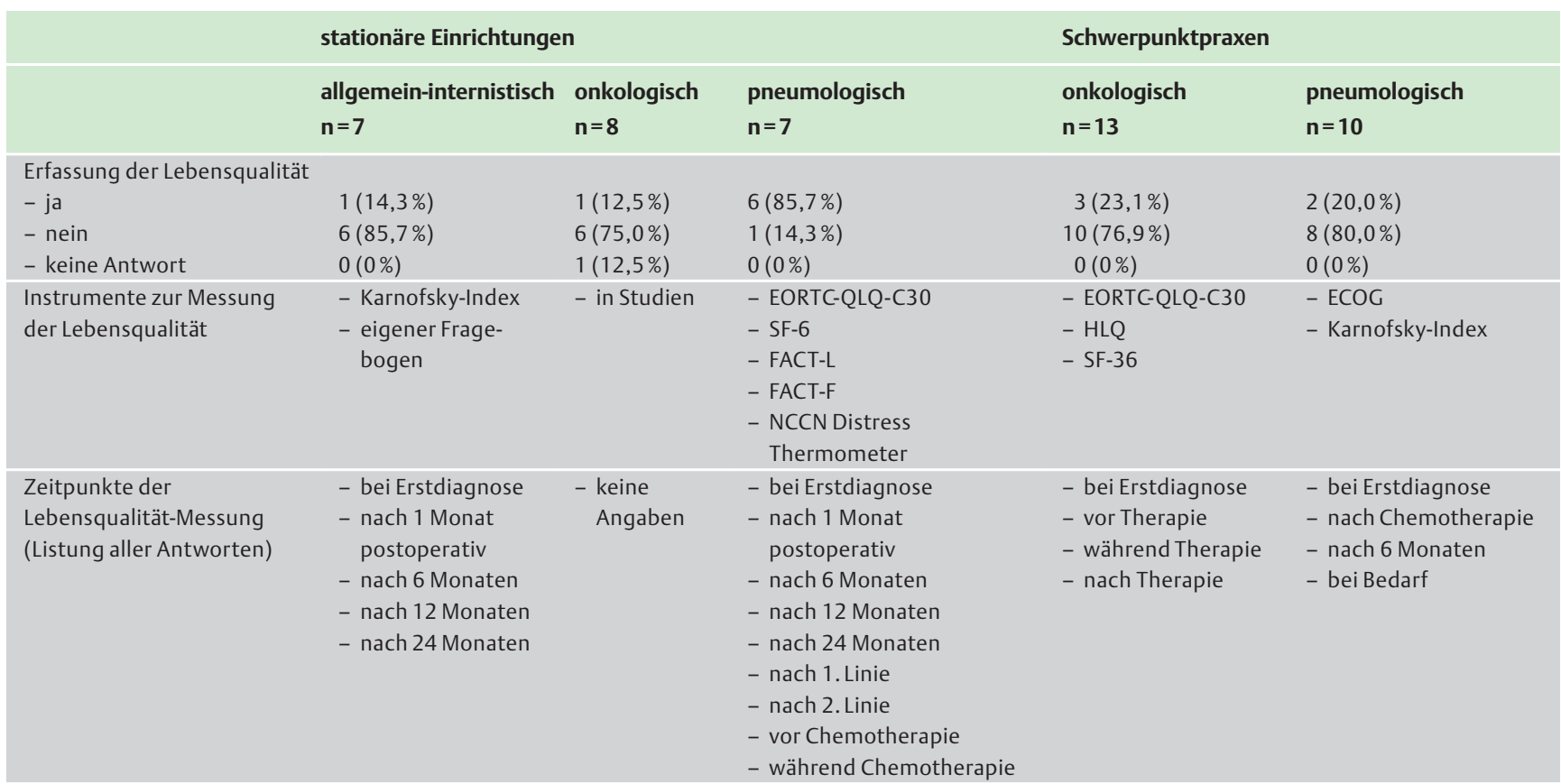

\section{Ergebnisse}

Eine Übersicht über angeschriebene Versorgungseinrichtungen sowie deren jeweilige Rücklaufquoten finden sich in $\bullet$ Tab. 1. Die Umfrageergebnisse werden detailliert in den Tab. 2 und - Tab. 3 wiedergegeben.

An dem Audit am 4.4.2011 nahmen Vertreter von elf Einrichtungen und eine Vertreterin der Selbsthilfegruppe Lungenkrebs e.V. teil. Nach einer ausführlichen Präsentation und anschließenden Diskussion der Ergebnisse erklärten sich alle Audit-Teilnehmer mit der Publikation der Ergebnisse in der vorliegenden Form einverstanden.

\section{Diskussion}

Unsere Umfrageergebnisse zeigten bei gutem Rücklauf eine nahezu vollständige konzeptionelle Leitlinientreue in stationären pneumologischen bzw. pneumologisch-thoraxchirurgischen Einrichtungen. Bei keinem Qualitätsindikator gab es wesentliche Abweichungen.

In stationären Einrichtungen mit onkologischem Schwerpunkt sowie pneumologischen bzw. onkologischen Schwerpunktpraxen ergaben sich Abweichungen bzw. Nichtbeachtungen von mehreren Qualitätsindikatoren der Leitlinie. Die Abweichungen betrafen v.a. multimodale Therapievorgaben mit einem höheren Therapie- und Koordinationsaufwand. Die tendenziell geringste Leitlinientreue wiesen Einrichtungen auf, die keine pneumologische oder onkologische Spezialisierung besaßen.

Unsere Untersuchung zur Implementierung der Leitlinie ergab somit Hinweise auf einen unterschiedlichen Umsetzungsgrad in Einrichtungen unterschiedlicher Spezialisierung. Letztendlich erlauben die erhobenen Daten aber insofern keine Aussage über die Behandlungsqualität der befragten Zentren, als lediglich Kennzahlen der Umsetzung, aber keine therapeutischen Endpunkte abgefragt wurden.
Die Leitlinie wurde insbesondere erstellt, um die Behandlungsqualität von Patienten außerhalb hochspezialisierter Einrichtungen mit trimodalen Versorgungsmöglichkeiten zu gewährleisten. Dieses Ziel ist ein Jahr nach Erscheinen der Leitlinien bei weitem noch nicht erreicht worden. Die Gründe für eine unzureichende Durchdringung wurden in dieser Untersuchung nicht abgefragt und sind nicht bekannt.

Allerdings ist bislang noch nicht erwiesen, ob beim Lungenkarzinom Leitlinientreue eine prognostische Relevanz besitzt. Beim Mammakarzinom hingegen konnte in einer Beobachtungsstudie für die Region Ulm gezeigt werden, dass sowohl rezidivfreies Überleben als auch Gesamtüberleben negativ mit der Anzahl der Abweichungen von Leitlinienempfehlungen korrelierte [6]. Solange für das Lungenkarzinom keine vergleichbaren Beobachtungstudien vorliegen, empfiehlt sich nichtsdestoweniger ein leitlinientreues Verhalten, denn es muss mindestens mit der Möglichkeit eines positiven prognostischen Einflusses gerechnet werden.

Noch heterogener waren die Ergebnisse im Hinblick auf die Erfassung der Lebensqualität. Methodisch einschränkend muss gesagt werden, dass das Ergebnis eine Mischung von Parametern zur Beschreibung des Allgemeinzustandes der Patienten bzw. ihrer Symptomlast und anerkannten Parametern zur Erfassung der Lebensqualität darstellt. Die Erfassung von Komorbiditäten wurde nicht erfragt. Weder national noch international existieren allerdings einheitliche Vorgaben, wie die Parameter zur Beschreibung von Lebensqualität, Symptomlast und Komorbiditäten bei Lungenkrebspatienten einheitlich und damit vergleichbar zu erfassen sind.

Bei diesem Qualitätsparameter zeigten ausschließlich stationäre pneumologische Einrichtungen eine zufriedenstellende Anwendungsquote.

Als möglicher Bias in dieser Studie ist prinzipiell die gewählte Methode der Selbstauskunft mit nur eingeschränkter Möglichkeit der Ergebnisvalidierung zu nennen [7]. Aus diesem Grunde führten wir ein Audit zur Ergebnispräsentation und Möglichkeit der kritischen Diskussion der Ergebnisse durch, das von rund 
einem Viertel der an der Studie partizipierenden Einrichtungen besucht wurde. Die Audit-Teilnehmer bestätigten die vorliegenden Ergebnisse.

Die Implementierung einer Leitlinie ist die wesentliche Phase nach deren Erstellungsprozess, entscheidet sie doch über deren späteren Anwendungs- und Umsetzungsgrad durch alle angesprochenen Fachdisziplinen in der alltäglichen Praxis [8-10]. Die deutsche S3-Leitlinie bietet mit den enthaltenen neun Qualitätsindikatoren, der Leitlinien-Kurzfassung sowie einem Patientenfragebogen nach beruflichen Ursachen von Lungentumoren Instrumente zur besseren Implementierung und späteren Umsetzung $[1,11,12]$.

International finden sich erste Studien, die sich der Frage nach dem Umsetzungsgrad von Behandlungsempfehlungen in Lungenkarzinom-Leitlinien annehmen. In diesen Arbeiten werden mitunter gravierende Abweichungen von Leitlinienempfehlungen in der Routinebehandlung von Lungenkrebspatienten aufgezeigt [13-21]. Derartige Studien sind eine wesentliche Grundlage für das Monitoring von Leitlinien in deren Umsetzungsphase und bieten so die Möglichkeit der späteren Optimierung. In einem durch den Europarat 2001 propagierten Modell wird Evaluation als ein integraler Bestandteil des Lebenszyklus von Leitlinien gesehen [10]. Für Deutschland gibt es jedoch bislang keine vergleichbaren Studien. Die vorliegende Arbeit stellt somit einen ersten Schritt bei der Beantwortung dieser Fragestellung auf einer regionalen Ebene in Deutschland dar.

Als nächste Schritte sollten gleichartige Umfragen flächendeckend über die regionalen Tumorzentren in allen Bundesländern durchgeführt werden. In einer zweiten Runde der Befragung müssen ergänzend die Gründe für Abweichungen von leitliniengerechten Behandlungskonzepten erfragt werden. Sinnvoll wäre eine zeitgleiche, koordinierte Vorgehensweise der deutschen Tumorzentren, um die bestmögliche Vergleichbarkeit zu erzielen.

\section{Interessenkonflikt}

$\nabla$

Die Autoren geben an, dass kein Interessenkonflikt besteht.

\section{Literatur}

1 Goeckenjan G, Sitter H, Thomas $M$ et al. Prävention, Diagnostik, Therapie und Nachsorge des Lungenkarzinoms. Pneumologie 2010; 64: e1 e164

2 Berliner Senatsverwaltung für Gesundheit. Fallzahlen operativer Krebsbehandlungen in Berliner Krankenhäusern. http://www.berlin. de (abgerufen am 28.02.2012)

3 Blum T, Schönfeld N, Kollmeier J et al. Lungenkrebs in Deutschland - zur Versorgungslage der Nation. Pneumologie 2011; 65: 7-18

4 Kassenärztliche Vereinigung Berlin. Ärzteverzeichnis. http://www. kvberlin.de (abgerufen am 28.02.2012)

5 Berliner Krankenhausgesellschaft. Berliner Krankenhausverzeichnis. http://www.berliner-krankenhausverzeichnis.de (abgerufen am 28.02.2012)

6 Schwentner $L$, Wolters $R$, Wischnewsky $M$ et al. Survival of patients with bilateral versus unilateral breast cancer and impact of guideline adher- ent adjuvant treatment: a multi-centre cohort study of 5292 patients. Breast 2012; 21: $171-177$

7 O'Toole BI. Survey subjects and the quality of health surveys. Med J Aust 1990; 152: 171-174

8 Arbeitsgemeinschaft der Wissenschaftlichen Medizinischen Fachgesellschaften. Ärztliches Zentrum für Qualität in der Medizin. Deutsches Instrument zur methodischen Leitlinien-Bewertung (DELBI). http:// www.leitlinien.de/leitlinienmethodik/leitlinienbewertung/delbi (abgerufen am 28.02.2012)

9 Brouwers MK, Browman GP, Burgers JS et al. for the AGREE Next Steps Consortium. AGREE II: Advancing guideline development, reporting and evaluation in healthcare. Can Med Assoc J 2010: DOI 10.1503/ cmaj.090449

10 Europarat. Entwicklung einer Methodik für die Ausarbeitung von Leitlinien für optimale medizinische Praxis. Empfehlung Rec(2001)13 des Europarates und Erläuterndes Memorandum. Deutschsprachige Ausgabe. Z Arztl Fortbild Qualitatssich 2002; 96: 5-59

11 Goeckenjan G, Sitter H, Thomas M et al. Prävention, Diagnostik, Therapie und Nachsorge des Lungenkarzinoms. Interdisziplinäre S3-Leitlinie der Deutschen Gesellschaft für Pneumologie und Beatmungsmedizin und der Deutschen Krebsgesellschaft-Kurzfassung. Pneumologie 2011; 65: e51-e75

12 Arbeitsgemeinschaft der Wissenschaftlichen Medizinischen Fachgesellschaften. Fragebogen nach beruflichen Ursachen bei Patienten mit Lungentumoren - S3-Leitlinie „Prävention, Diagnostik, Therapie und Nachsorge des Lungenkarzinoms“, Anhang zu Kapitel 2.7. http:// www.awmf.org/leitlinien/detail/11/020-007.html (abgerufen am 28.02.2012)

13 Charloux A, Brunelli A, Bolliger CT et al. Lung function evaluation before surgery in lung cancer patients: how are recent advances put into practice? A survey among members of the European Society of Thoracic Surgeons (ESTS) and of the Thoracic Oncology Section of the European Respiratory Society (ERS). Interact Cardiovasc Thorac Surg 2009; 9: $925-931$

14 Eisenberg RL, Bankier AA, Boiselle PM. Compliance with Fleischner Society guidelines for management of small lung nodules: a survey of 834 radiologists. Radiology 2010; 255: $218-224$

15 Feely MA, Hartman TE. Inappropriate application of nodule management guidelines in radiologist reports before and after revision of exclusion criteria. AJR Am J Roentgenol 2011; 196: 1115-1119

16 Couraud S, Fournel P, Moro-Sibilot D et al. Are clinical guidelines applied in routine daily practice?: a French regional survey of physicians' clinical practices in lung cancer management (EPOTRA). Clin Lung Cancer 2011; $12: 298-306$

17 Salloum RG, Smith TJ, Jensen GA et al. Factors associated with adherence to chemotherapy guidelines in patients with non-small cell lung cancer. Lung Cancer 2012; 75: 255-260

18 Heijmen L, van Laarhoven HW, Punt CJ et al. Encouraging results in older patients receiving chemotherapy: a retrospective analysis of treatment guideline adherence in daily practice. Geriatr Gerontol Int 2012; $12: 80-85$

19 Baughan P, Keatings J, O'Neill B. Urgent suspected cancer referrals from general practice: audit of compliance with guidelines and referral outcomes. Br J Gen Pract 2011; 61: e700 - 706

20 Jakobsen E, Palshof T, Osterlind $K$ et al. Data from a national lung cancer registry contributes to improve outcome and quality of surgery: Danish results. Eur J Cardiothorac Surg 2009; 35: 348 - 352

21 Sleeman D, Moss L, Gyftodimos $E$ et al. A comparison between clinical decisions made about lung cancer patients and those inherent in the corresponding Scottish Intercollegiate Guidelines Network (SIGN) guideline. Health Informatics J 2010; 16: 260-273 